\title{
Lateral composition modulation in AIAs/InAs and GaAs/InAs short period superlattices structures: The role of surface segregation
}

\author{
C. Dorin and J. Mirecki Millunchick \\ Department of Materials Science and Engineering, University of Michigan, Ann Arbor, \\ Michigan 48109-2136
}

(Received 28 June 2001; accepted for publication 28 September 2001)

\begin{abstract}
The effect of In surface segregation on the microstructure of short period superlattices (SPSs) in two different material systems with nominally equivalent lattice misfit, AlAs/InAs and GaAs/InAs, has been investigated and compared. It was found that the quality of the SPSs and the appearance of lateral composition modulation are remarkably different in these two systems. For AlAs/InAs SPSs grown at temperatures of $T=500{ }^{\circ} \mathrm{C}$, uniform structures devoid of lateral composition modulation were obtained. Samples grown at $T>500^{\circ} \mathrm{C}$ exhibit lateral composition modulation. Uniform and homogeneous SPS structures were not obtained in the GaAs/InAs structures over the entire temperature range examined in this study $\left(475^{\circ} \mathrm{C} \leqslant T \leqslant 510^{\circ} \mathrm{C}\right)$. Instead, lateral composition modulation with varying degrees of regularity was always observed. It was found that In segregation and roughening determine the microstructure. A kinetic exchange model predicts that at an optimum temperature the SPS layers are more intermixed for the AlAs/InAs SPSs. Thus, the lattice mismatch is lower and the driving force for roughening is reduced, resulting in uniform SPS structures. Growing the structure away from the optimum temperature for smooth growth may induce roughening-driven composition modulation. The GaAs/InAs structures are less intermixed over the temperatures studied, which results in higher mismatch between the individual layers and a higher driving force for roughening and lateral composition modulation. (C) 2002 American Institute of Physics. [DOI: 10.1063/1.1421240]
\end{abstract}

\section{INTRODUCTION}

The alloying of compound semiconductors allows band gap engineering and thus optical properties can be tailored for specific device applications. Typically, it is assumed that alloys of this sort are fully random. That is, there is no correlation among the individual alloy species on the crystalline lattice. However, experiments show that phase separation and/or ordering of the alloy is possible under certain growth conditions. ${ }^{1}$ Although phase separation in particular is generally avoided in device structures, the recent advent of nanotechnology has spurred an effort to exploit it for quantum confinement devices., ${ }^{2,3}$

Composition modulation is a type of phase separation, and refers specifically to the formation of phase separated, self-organized periodic structures. Virtually all semiconductor alloys have been observed to undergo spontaneous composition modulation under a variety of growth conditions. Vertical composition modulation has been observed in InAsSb layers deposited at low temperatures. ${ }^{4-6}$ For example, In $\mathrm{As}_{0.5} \mathrm{Sb}_{0.5}$ grown at $430^{\circ} \mathrm{C}$ produced a random alloy, while the same material grown at $340^{\circ} \mathrm{C}$ was strongly modulated along the (001) growth direction. This phenomenon has been attributed to the nucleation of different phase islands at the growth front. ${ }^{6}$

Composition modulation often occurs during the deposition of alloys, as in InAsSb, but also occurs as a result of the deposition of structures such as short period superlattices (SPS) where each layer of the superlattice is on the order of a few monolayers. There has been a great deal of recent progress in a number of III-V alloy systems in this area of research. ${ }^{3-16}$ For example, AlAs/InAs SPS structures deposited on InP (001) were shown to exhibit composition modulation perpendicular to the growth direction, and the modulation profile was correlated to surface undulation present in the film. ${ }^{10}$ Cross-sectional transmission electron micrographs (XTEM) in the [1 10$]$ projection show a modulation wavelength along the [110] direction that was found to vary between $15<\Lambda<25 \mathrm{~nm}$. Lateral composition modulation was also observed in XTEM micrographs in the [110] projection where the modulation was along the [1 10$]$ with wavelength of the order of $\Lambda \approx 33 \mathrm{~nm} .{ }^{10}$ Scanning TEM has shown that the In composition within the In-rich and Al-rich regions is $x_{\text {In rich }}=0.76$ and $x_{\mathrm{Al} \mathrm{rich}}=0.38$, respectively. ${ }^{12}$ The modulation direction has been show to vary with the average composition $\bar{x}$ of the SPS. ${ }^{7}$ For structures where $\bar{x}$ is tensile $(\bar{x}$ $\langle 0.53)$, the modulation is along $\langle 310\rangle$ directions. For structures where $\bar{x}$ is compressive $(\bar{x}>0.53)$, the modulation is along the elastically soft $\langle 100\rangle$ directions. This effect is postulated to be a result of competition between alternating compressive and tensile morphologies. The modulation direction can be chosen by performing growths on offcut substrates. ${ }^{13}$ Follstaedt $e$ t al. found that the growth of InAs/ AlAs SPSs on appropriately miscut InP substrates alters the microstructure of composition modulation from a twodimensional (2D) organization of wires to a single dominant modulation direction with wire lengths up to $\sim 1 \mu \mathrm{m} .{ }^{13}$

Lateral composition modulation has also been observed in SPS structures of InAs/GaAs on InP (001) substrates and 
TABLE I. Summary of the material system, growth temperature $T$, growth rate $R$, average In SPS composition $\bar{x}$, and lateral wavelength $\Lambda$ along both the [110] and [1 $\overline{1} 0]$ directions for the samples examined in this study.

\begin{tabular}{ccccccc}
\hline \hline $\begin{array}{c}\text { Sample } \\
\text { No. }\end{array}$ & Material system & $\begin{array}{c}T \\
\left({ }^{\circ} \mathrm{C}\right)\end{array}$ & $\begin{array}{c}R \\
(\mathrm{ML} / \mathrm{s})\end{array}$ & $\bar{x}$ & {$[110]$} & \multicolumn{2}{c}{$\Lambda(\mathrm{nm})$} \\
\cline { 5 - 7 } A450 & AlAs/InAs & 450 & 0.48 & 0.45 & N/A & N/A \\
A500 & AlAs/InAs & 500 & 0.59 & 0.46 & N/A & N/A \\
A515 & AlAs/InAs & 515 & 0.42 & 0.44 & 29 & 44 \\
A520 & AlAs/InAs & 520 & 0.42 & 0.44 & 17 & 26 \\
G475 & GaAs/InAs & 475 & 0.48 & 0.48 & 15 & 26 \\
G480 & GaAs/InAs & 480 & 0.48 & 0.48 & 23 & 25 \\
G485 & GaAs/InAs & 485 & 0.48 & 0.48 & 17 & 17 \\
G490 & GaAs/InAs & 490 & 0.48 & 0.48 & 31 & 71 \\
G500 & GaAs/InAs & 500 & 0.48 & 0.48 & 27 & 93 \\
G510 & GaAs/InAs & 510 & 0.48 & 0.48 & N/A & 14 \\
\hline \hline
\end{tabular}

InP/GaP on GaAs (001) substrates, however, for these materials the modulation was reported to occur only in the [1 10$]$ direction. ${ }^{14}$ It is believed that this orientation is chosen as a result of fast diffusion along dimer rows in the that direction, thus forming sheets of alternately In-rich and Ga-rich regions that are roughly $30 \mathrm{~nm}$ wide and several micrometers long. ${ }^{15}$

Although the formation of lateral composition modulation is not fully understood, several models have been proposed. A common explanation for composition modulation in both the lateral and vertical directions is spinodal decomposition. However, this explanation is unlikely since the growth temperature is much higher than the critical temperature for spinodal decomposition calculated by Glas. ${ }^{17}$ Cheng and co-workers ${ }^{16}$ have developed a model referred to as straininduced lateral ordering (SILO) to explain how lateral composition modulation in SPSs is initiated. In most cases, composition modulation has been observed in SPSs in which the constituent binary alloys are strain balanced to the InP substrate, i.e., InAs and GaAs are both roughly $3.5 \%$ lattice mismatched with respect to the InP substrate, except they are opposite in sign. Despite the rather substantial misfit between each individual layer, a SPS consisting of a few monolayers of each binary compound can be made to be strain balanced with zero global strain. Cheng et al. observed that composition modulation occurred when the SPS is deviates slightly from this condition and the resulting two-dimensional (2D) islands produce strain fields at the surface and initiate composition modulation. ${ }^{14}$

It has been suggested that undulations at the growth front initiate lateral composition modulation, ${ }^{10}$ implying that surface instabilities play a major role in this phenomenon. ${ }^{18}$ In fact, several models predict that stress-driven morphological undulations are coupled to compositional modulations. ${ }^{19-21}$ For example, Spencer et al. found that compositional stresses in alloy films further destabilize morphological instabilities that arise due to misfit stresses. ${ }^{20}$ These models, however, consider the growth of single alloy layers and may not be valid for multilayer structures. Shilkrot, Srolovitz, and Tersoff ${ }^{21}$ applied linear stability analysis to multilayer structures. They found that roughening of the surface depends greatly on the stress contributions from the buried interfaces. Under certain conditions, this model predicts that the multilayer structure exhibits lateral composition modulation as a result of variations in the thickness of the individual layer thicknesses as opposed to alloy decomposition.

Despite all of the recent work in this area, a comprehensive model for lateral composition modulation has yet to emerge. In this article, we compare lateral composition modulation in AlAs/InAs and GaAs/InAs SPS structures grown at various growth temperatures. Although AlAs and GaAs have similar lattice constants, the microstructure of the phase separation is significantly different in the two material systems. In surface segregation is shown to be the cause of these differences.

\section{EXPERIMENT}

All growths were carried out in an EPI 930 molecular beam epitaxy chamber with solid sources for $\mathrm{ln}, \mathrm{Ga}$, and $\mathrm{Al}$ and a valved cracking cell for As. The films were grown upon InP (001) substrates that were prepared by heating to $500{ }^{\circ} \mathrm{C}$ under an $\mathrm{As}_{4}$ overpressure to remove the oxide layer. In all cases, a nominally lattice matched $\operatorname{In}_{0.53} \mathrm{Ga}_{0.47} \mathrm{As}$ random alloy was grown to a thickness on the order of $500 \mathrm{~nm}$ at a growth rate $0.42<R<0.59$ monolayer(ML)/s calibrated by reflection high energy electron diffraction (RHEED) oscillations, and at a growth temperature of $T=500{ }^{\circ} \mathrm{C}$ that was monitored by an optical pyrometer. Following buffer layer growth, the SPS was grown by depositing $2 \mathrm{ML}$ of InAs followed by the same amount of either AlAs or GaAs and repeated 100 times. Table I lists the samples grown in this study and the growth conditions for each. Finally, the structure was capped with latticed matched InGaAs in order to prevent oxidation of the Al-containing films.

The samples were examined via a battery of in situ and ex situ characterization tools. The quality of the crystalline surface was monitored in situ by RHEED. X-ray diffraction (XRD) $\theta / 2 \theta$ scans and reciprocal space maps were recorded with $\mathrm{Cu} K \alpha$ radiation in order to characterize the microstructure of the SPS. XTEM was performed on representative samples to corroborate the XRD data. Samples were prepared using mechanical thinning, followed by Ar-ion milling at $4.5 \mathrm{kV}$ and at angles between $3^{\circ}$ and $5^{\circ}$. The buildup of In-metal islands on the surface was confined to the substrate 


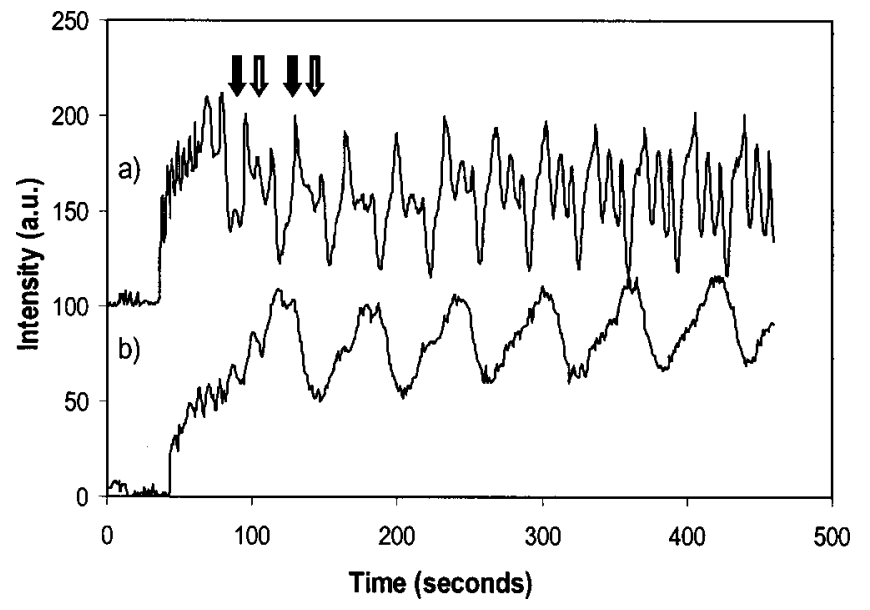

FIG. 1. Evolution of the RHEED specular spot as a function of time for AlAs/InAs SPSs grown at $T=$ (a) $500{ }^{\circ} \mathrm{C}$ and (b) $520{ }^{\circ} \mathrm{C}$ (solid arrow denotes InAs deposition and open arrow denotes AlAs).

and did not affect the sample quality in the region of interest. XTEM studies were carried out on a JEOL 4000EX microscope operated at $400 \mathrm{kV}$.

\section{RESULTS}

AlAs/InAs and GaAs/InAs SPS structures have similar lattice parameters, however, the resulting microstructures are very different. This study was conducted to compare the two systems in order to identify the mechanisms that initiate lateral composition modulation. Both materials systems exhibit lateral composition modulation under the appropriate growth conditions.

\section{A. AIAs/InAs SPS structures}

It was found that the growth temperature was a significant factor in the final microstructure of compositionally modulated samples. For the lowest temperatures studied, $T$ $=450{ }^{\circ} \mathrm{C}$ (sample A450) and $T=500{ }^{\circ} \mathrm{C}$ (sample A500), the growth proceeded via the layer-by-layer growth mode. Figure 1 shows plots of the intensity of the specular reflection of the RHEED beam as a function of time for the InAs/AlAs SPS structures grown at $T=500$ and $520^{\circ} \mathrm{C}$. At the beginning of growth, several monolayers of InGaAs buffer layer were deposited. RHEED oscillations arising from this growth are clearly observed. Immediately following, the individual SPS layers were deposited, denoted by the arrows in Fig. 1. Four oscillations per period are clearly observed for sample A500, indicating that growth proceeds via the layerby-layer mode [Fig. 1(a)]. The surface reconstruction was $(2 \times 1)$ during the $I n A s$ deposition and $(3 \times 1)$ during the AlAs deposition and the final RHEED pattern was streaky, indicative of a smooth surface. The samples grown at higher temperature, $T=515^{\circ} \mathrm{C}$ (sample A515) and $T=520^{\circ} \mathrm{C}$ (sample A520), on the other hand, did not grow via the layerby-layer growth mode, as noted by the absence of growth oscillations. Oscillations in the RHEED spectral intensity that are present in Fig. 1(b) arise due to changes in the surface reconstruction from $(2 \times 1)$ during InAs growth and $(3 \times 1)$ during AlAs growth, the same as for the lower tem-

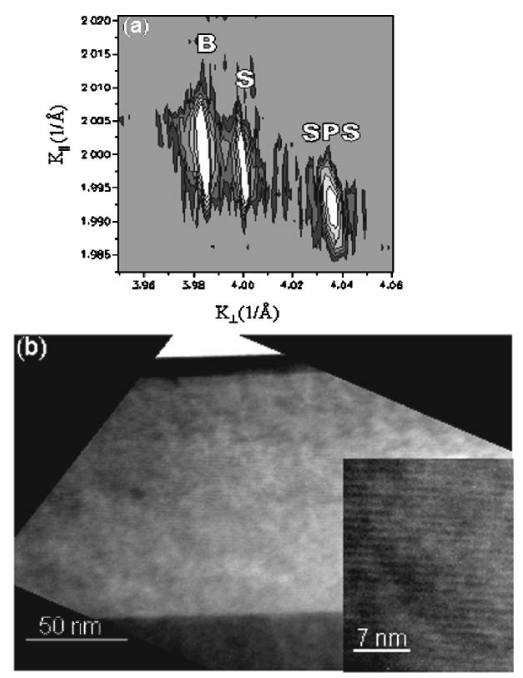

FIG. 2. (a) (224) XRD reciprocal space map for sample A500. Peaks are observed that correspond to the substrate (S), buffer layer (B), and SPS layer (SPS). (b) (110) bright field XTEM image of this sample. The inset is a higher magnification image that shows the individual SPS layers.

perature growth. The final RHEED pattern for this sample was somewhat spotty, which implies that some degree of surface roughening has occurred. These results are corroborated with scanning tunneling microscopy (STM) images that show that SPSs grown at low temperatures are planar and SPSs grown at higher temperatures are rougher with a high density of $2 \mathrm{D}$ islands. ${ }^{22}$

XRD and XTEM were performed to learn about the SPS microstructure as a function of the growth temperature. Lateral satellites in reciprocal space have been demonstrated to be a signature of composition modulation. ${ }^{23}$ The (224) x-ray diffraction reciprocal space map for sample A500 [Fig. 2(a)] shows peaks from the substrate, buffer layer, and the Bragg peak of the SPS. The XRD of sample A450 is similar to that of A500. Figure 2(b) is a bright field image of the (110) zone axis for sample A500 and shows the individual SPS layers without any visible defects or lateral inhomogeneities, in agreement with the XRD data.

The reciprocal space maps of the samples grown at higher temperatures exhibit lateral satellites that arise from periodicity in the growth plane. Figures 3(a) and 3(b) are (224) and $(2 \overline{2} 4)$ x-ray diffraction reciprocal space maps of sample A520 that show peaks from the substrate, buffer layer, and the Bragg peak of the SPS in addition to satellites that arose from lateral composition modulation in both the [110] and [1 $1 \overline{1} 0]$ directions. Lateral composition modulation of this sample is confirmed by TEM. Figures 3(c) and 3(d) are dark field cross-sectional TEM images for the (002) reflection for the [110] and [1 $1 \overline{1} 0]$ projections for sample A520 that show strong regular and periodic lateral contrast that is characteristic of lateral composition modulation. The lateral composition modulation of this sample has wavelengths of $\Lambda_{[1 \overline{1} 0]} \sim 26 \mathrm{~nm}$ and $\Lambda_{[110]} \sim 17 \mathrm{~nm}$.

A $\theta / 2 \theta$ scan about the (004) diffraction condition provides information regarding the vertical regularity of the SPS structure. The presence of satellite peaks at $\Delta \theta \approx \pm 4.2^{\circ}$ indicates the presence of a well-defined regular SPS with ver- 

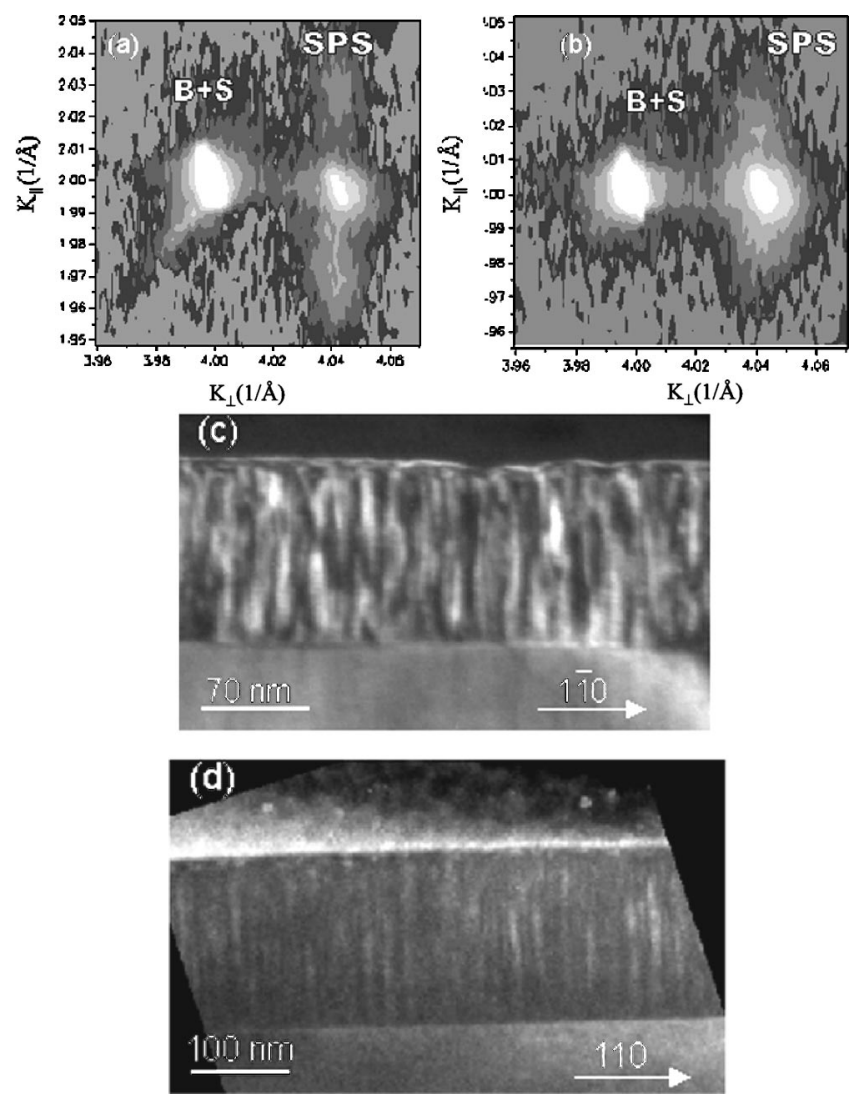

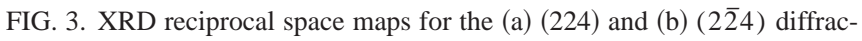
tion conditions for sample A520. The maps show lateral satellites due to lateral composition modulation with wavelengths $\Lambda_{[1 \overline{1} 0]}=26 \mathrm{~nm}$ and $\Lambda_{[110]}=17 \mathrm{~nm}$. (002) XTEM dark field images of the (c) [1 10$]$ and (d) [110] projections for the same sample.

tical periodicity of 4 ML. The $\theta / 2 \theta$ scans (Fig. 4) of samples $\mathrm{A} 450, \mathrm{~A} 500, \mathrm{~A} 515$, and A520 show that first order SPS vertical satellites are present in these films. Samples A450 and A500 have relatively strong vertical satellites, implying that the structure is well defined in the vertical direction. The higher temperature samples have significantly weaker satellites, suggesting that the vertical periodicity is less coherent in these samples. This is consistent with the reciprocal space and TEM data that show that the higher $T$ samples are modu-

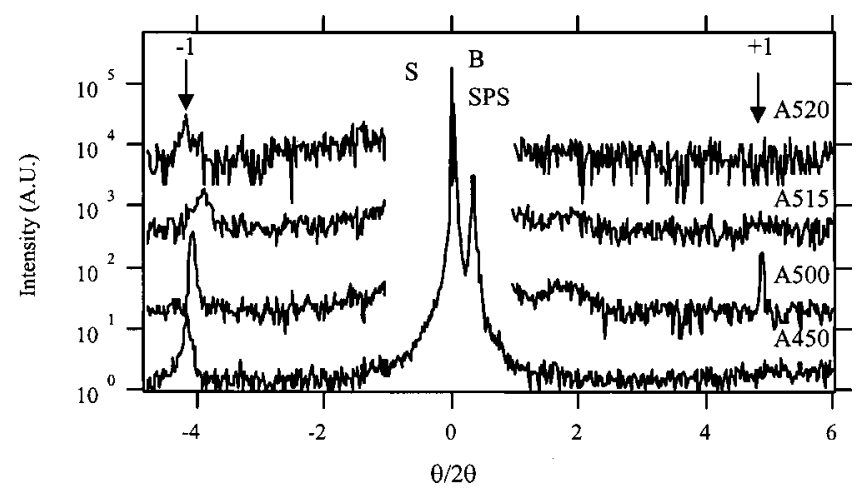

FIG. 4. (004) $\theta / 2 \theta$ XRD scans showing peaks due to the buffer layer (B), substrate (S), SPS, and first-order SPS vertical satellites $( \pm 1)$ for samples $\mathrm{A} 450, \mathrm{~A} 500, \mathrm{~A} 515$, and A520.
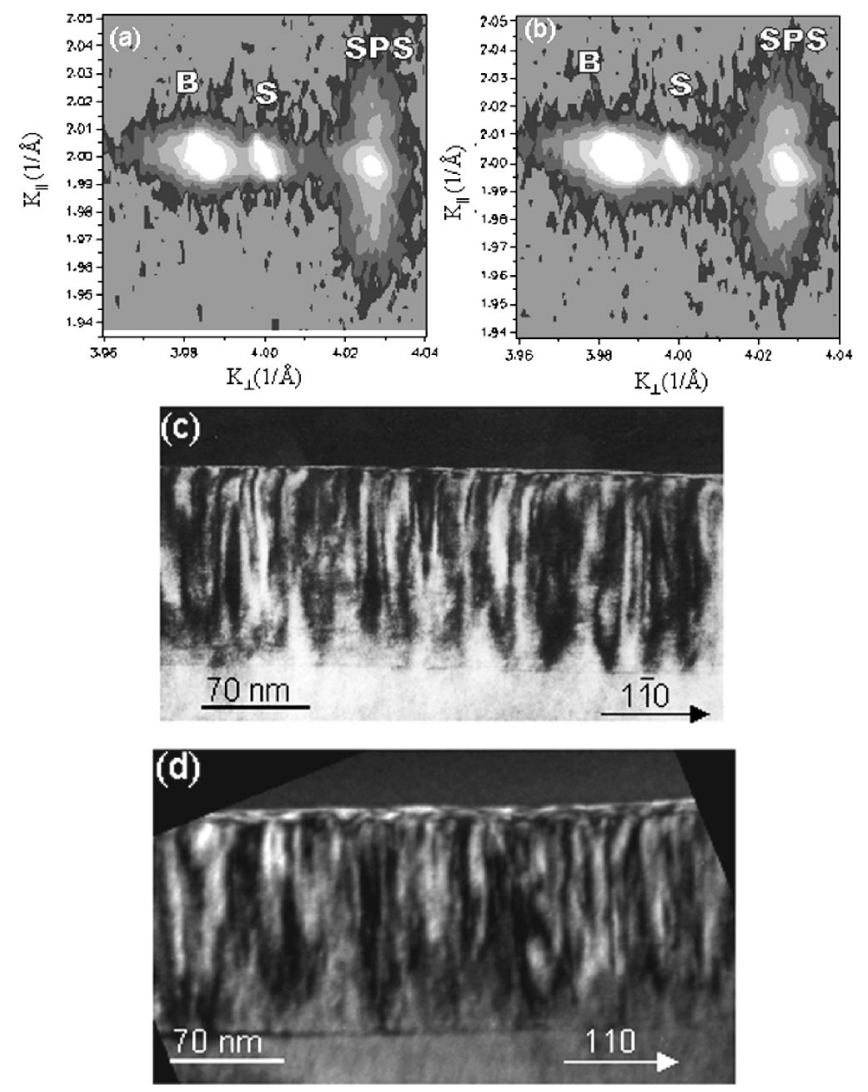

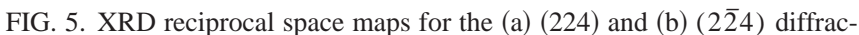
tion conditions for sample G490. The maps show lateral satellites due to lateral composition modulation with wavelengths $\Lambda_{[1 \overline{1} 0]}=71 \mathrm{~nm}$ and $\Lambda_{[110]}=31 \mathrm{~nm}$. (002) XTEM dark field images in the (c) [1 10$]$ and (d) [110] projections for the same sample.

lated in the lateral direction (Fig. 3). That is, lateral composition modulation necessarily destroys the vertical periodicity of the SPS structure.

\section{B. GaAs/InAs SPS structures}

Lateral composition modulation was observed in all the GaAs/InAs SPS structures examined in this study. Furthermore, roughening was also observed to some degree in these
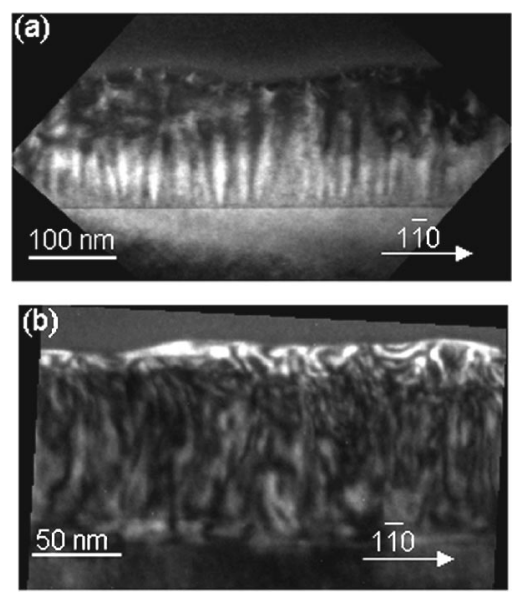

FIG. 6. (002) XTEM dark field images for samples (a) G480 and (b) G510. 

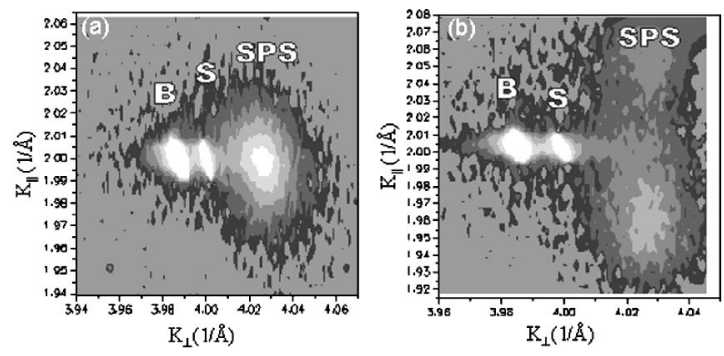

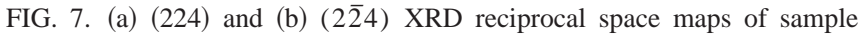
G510.

samples. The surface reconstruction was always $(2 \times 1)$ during the InAs deposition and $(3 \times 1)$ during the GaAs deposition. The RHEED streaks began to modulate in intensity along their length, indicating surface roughening of the samples grown at $485^{\circ} \mathrm{C} \leqslant T \leqslant 490^{\circ} \mathrm{C}$. Figure 5 shows XRD reciprocal space maps and XTEM images of the GaAs/InAs SPS sample grown at $T=490{ }^{\circ} \mathrm{C}$ (sample G490). These data show that the sample possesses very regular, robust lateral composition modulation along both [110] and [1 $\overline{1} 0]$ directions with modulation wavelengths of $\Lambda_{[110]}=31 \mathrm{~nm}$ and $\Lambda_{[1 \overline{1} 0]}=71 \mathrm{~nm}$.

For samples grown at $T<485^{\circ} \mathrm{C}$ the RHEED pattern became spotty, indicative of three-dimensional (3D) island formation, after nominally half the SPS was deposited. Figure 6(a) shows a XTEM dark field micrograph of a GaAs/ InAs SPS grown at $T=480^{\circ} \mathrm{C}$ (G480). Near the buffer layer SPS interface, strong, regular lateral composition modulation is observed. However, that periodicity is destroyed after approximately 45 cycles were deposited. The remainder of the film has irregular phase separation and a rough surface. Sample G475 (not shown) which was deposited at $T$ $=475^{\circ} \mathrm{C}$ behaves in a similar manner.

At $T>490^{\circ} \mathrm{C}$, the RHEED pattern also quickly degraded to spots during growth. Figure 6(b) is a XTEM dark field image of a GaAs/InAs SPS grown at $T=510^{\circ} \mathrm{C}$ (sample G510). In this image, lateral composition modulation is present, as indicated by the variation in contrast. However, the nature of the periodicity is considerably different than that in the lower temperature samples. In this case, the lateral contrast is not correlated from the top to the bottom of the film. This meandering of the phase separated regions is most likely related to the $3 \mathrm{D}$ islands that are present on the surface during growth, as indicated by the spotty RHEED pattern at the end of the deposition. It is interesting to note that the lateral composition modulation for sample G510 is only along the [1 $1 \overline{1} 0]$ direction as indicated by XRD reciprocal space maps (Fig. 7). The $\theta / 2 \theta$ XRD scan of this sample did not reveal the presence of SPS vertical satellites, indicating that the SPS is disordered.

\section{DISCUSSION}

The appearance of lateral composition modulation has been correlated to surface undulations. ${ }^{9}$ Therefore, it is reasonable to consider that surface diffusion is an important mechanism by which this microstructure forms. As a result, it is expected that the lateral periodicity should increase with

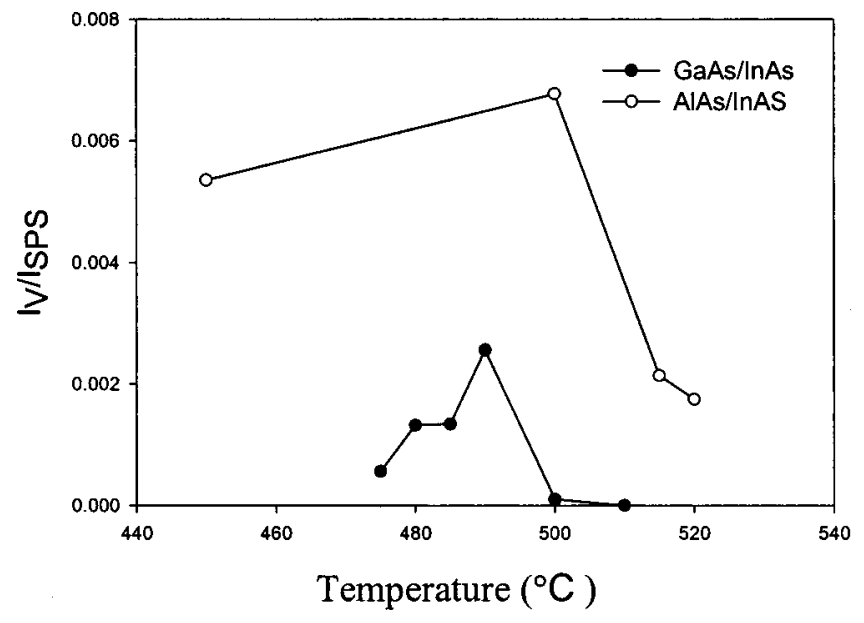

FIG. 8. Plot of the relative intensity of the first-order vertical satellites $I_{v} / I_{\mathrm{SPS}}$ as a function of the growth temperature.

an increase in growth temperature. It is also known that $\mathrm{Ga}$ atoms on GaAs have a lower activation energy to diffuse than $\mathrm{Al}$ atoms on AlAs. ${ }^{24}$ Therefore, the lateral periodicity of the GaAs-containing samples is expected to be larger since Ga has a longer diffusion length compared to Al. Line scans of the lateral satellites in the $\mathrm{x}$-ray reciprocal space maps were fitted with a Gaussian function to determine their position with respect to the SPS Bragg peak and to calculate the lateral periodicity of composition modulation $\Lambda$. $\Lambda$ of the AlAs/InAs and GaAs/InAs structures was found to be in the range of $15 \mathrm{~nm}<\Lambda<31 \mathrm{~nm}$ along the [110] direction and $17 \mathrm{~nm}<\Lambda<93 \mathrm{~nm}$ along the [1 10$]$ direction (see Table I). $\Lambda$ is not considerably temperature dependent in either $\langle 110\rangle$ direction, in agreement with other published reports. ${ }^{25}$ Despite the difference in diffusion lengths, there is no apparent material dependence on $\Lambda$. In addition, the strength of lateral composition modulation, which can be inferred from the intensity of the lateral satellites, is not strongly temperature nor material dependent.

It has been postulated that the lattice mismatch between the individual SPS layers is a mechanism for the appearance of lateral composition modulation. ${ }^{14}$ Therefore, it is surprising that the microstructure of the AlAs/InAs and GaAs/InAs structures is so different considering that the lattice parameters of AlAs and GaAs are nearly the same. One common theme in all of the samples studied is that some degree of roughening is correlated with the appearance of lateral composition modulation. ${ }^{22}$ For example, sample A500 exhibited layer-by-layer growth but no composition modulation (Fig. 2). Sample A520 became rough during growth and exhibited lateral composition modulation (Fig. 3). This roughening mechanism necessarily destroys the vertical periodicity of the SPS structure. Figure 8 shows a plot of the ratio of the intensity of the vertical satellites that arise due to the presence of the SPS relative to the intensity of the SPS Bragg peak $I_{v} / I_{\mathrm{SPS}}$, measured by $\theta / 2 \theta$ scans about the (004) reflection as a function of the growth temperature of both GaAs/ InAs and AlAs/InAs structures. $I_{v} / I_{\text {SPS }}$ decreases with an increase in temperature for the AlAs/InAs samples when $T$ $\geqslant 500{ }^{\circ} \mathrm{C}$, and for $\mathrm{GaAs} / \mathrm{InAs}$ samples when $T \geqslant 490{ }^{\circ} \mathrm{C}$. 

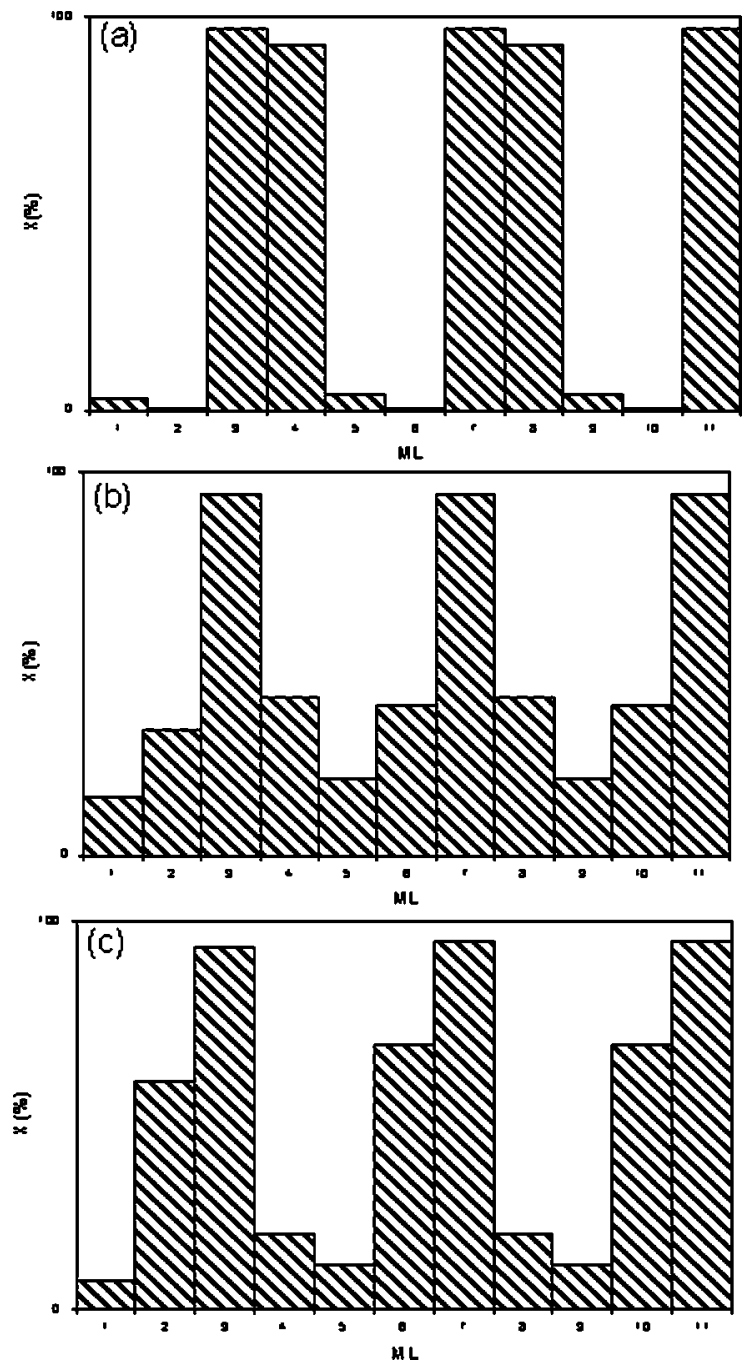

FIG. 9. In concentration profiles at $T=$ (a) 400 , (b) 500 , and (c) $600{ }^{\circ} \mathrm{C}$ for a SPS structure using $E_{1}=2 \mathrm{eV}$ and $E_{s}=0.25 \mathrm{eV}$.

This is consistent with the view that interfacial roughening increases with an increase in the growth temperature. The fact that $I_{v} / I_{\mathrm{SPS}}$ increases as $T$ increases for $T<500^{\circ} \mathrm{C}$ in the AlAs/InAs structures and for $T<490^{\circ} \mathrm{C}$ in the GaAs/ InAs structures cannot be understood by considering kinetic roughening alone. Instead, another mechanism must be operating in this temperature regime.

The difference in microstructure between the AlAs/InAs and $\mathrm{GaAs} / \mathrm{InAs}$ structures may be understood by considering the effect of In surface segregation during growth. In surface segregation refers to the preferential exchange of In atoms in the bulk to the surface. A kinetic model that assumes segregation is due only to the exchange process of In atoms between a bulk layer and a surface layer ${ }^{26}$ can be used to explain this behavior. The evolution of the number of In surface atoms is given by the balance of incoming and outgoing In atoms. The exchange process of In atoms from the bulk to the surface is achieved by overcoming an energy barrier $E_{1}$ with a rate of $\nu \exp \left(-E_{1} / k T\right)$, where $\nu$ is a vibration frequency on the order of $10^{13} \mathrm{~s}^{-1}, k$ is the Boltzmann constant, and $T$ is the growth temperature. A reverse exchange (from the surface to the bulk) is also possible. That

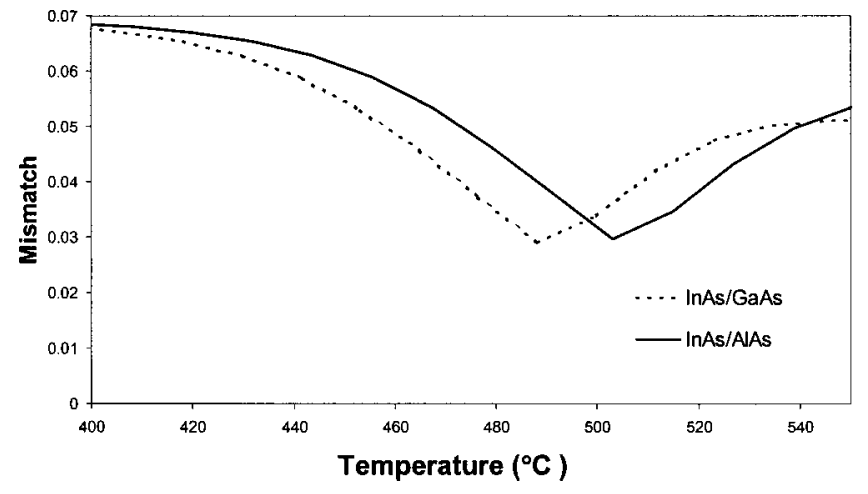

FIG. 10. Lattice mismatch between the In-rich and the $\mathrm{Ga}$ (or $\mathrm{Al}$ )-rich layers in the GaAs/InAs and AlAs/InAs SPS structures as a function of the growth temperature using the following parameters: $E_{1}^{\mathrm{Ga}}=2.04 \mathrm{eV}, E_{s}^{\mathrm{Ga}}=0.23 \mathrm{eV}$, $E_{1}^{\mathrm{Al}}=2.08 \mathrm{eV}$, and $E_{s}^{\mathrm{Al}}=0.29 \mathrm{eV}$.

energy barrier is $E_{2}=E_{1}+E_{s}$, where $E_{s}$ is the segregation energy, and has a rate of $\nu \exp \left(-E_{2} / k T\right)$. Assuming that segregation is due only to this exchange process, the number of In surface atoms is given by the equality of the incoming and outgoing In atoms, conserving the number of In atoms and total surface atoms as a function of time. ${ }^{26}$ The In concentration profile can be built by numerically solving these expressions. The concentration profile of a SPS at three different temperatures and for $E_{1}=1.75 \mathrm{eV}$ and $E_{s}=0.2 \mathrm{eV}$ is shown in Fig. 9. At low temperatures [Fig. 9(a)] the rate of In exchange is sufficiently slow there is no effective In segregation. This means that at this temperature each SPS individual layer is essentially pure InAs and GaAs (or AlAs). Because the lattice mismatch between GaAs (or AlAs) and InAs is high $(f=7 \%)$ the critical thickness for 3D island formation is small $(\approx 1 \mathrm{ML})$. In this case, 3D island formation will occur after the critical thickness of the overall structure is exceeded and will result in roughening the remainder of the film, like in sample G480 [Fig. 6(a)]. At intermediate temperatures [Fig. 9(b)], the SPS individual layers intermix due to In segregation, and the composition of each layer is more diluted. The lattice mismatch of these samples is lower and consequently the critical thickness for roughening is higher. The magnitude of the intermixing will determine whether the SPS remains well ordered, like in sample A500 (Fig. 2), or modulated, like in the sample G490 (Fig. 5). At even higher growth temperatures [Fig. 9(c)] the concentration profile is shifted by $1 \mathrm{ML}$ and the composition of each individual layer is again close to that of pure GaAs (or AlAs) and InAs, respectively. The fact that the concentration profile has shifted is not unexpected. As the temperature increases, the rate of In exchange to the surface is very high. At the InAs/GaAs interface, for example, a very large fraction of In will exchange with $\mathrm{Ga}$, effectively converting it to a GaAs/ InAs interface. Subsequent deposition of GaAs will again result in this exchange. The mismatch between the individual layers is high and results in more roughening. This is in agreement with the microstructure observed for sample G510 [Fig. 6(b)].

Figure 10 shows the average calculated lattice mismatch between the In-rich and $\mathrm{Ga}$ (or Al)-rich SPS layers versus temperature for $\mathrm{GaAs} / \mathrm{InAs}$ and AlAs/InAs SPS structures 
taking only In segregation into account. The values for $E_{1}$ were fit to our observations. In general, the model predicts that there is a minimum in the misfit at intermediate temperatures, the value of which depends sensitively on $E_{1}$. The value of $E_{s}$ determines the amount of intermixing. That is, as the value of $E_{s}$ increases, more In segregates to the surface. For Al-containing samples, no lateral composition modulation is observed at $T=500^{\circ} \mathrm{C}$. Furthermore, the relative SPS satellite intensity is highest for this sample (Fig. 8). Using this temperature to set the minimum $T, E_{1}=2.04 \mathrm{eV}$ is obtained. This is in reasonable agreement with other work that assumes $E_{1}$ to be of the order of the binding energy of AlAs. ${ }^{27}$ The positions of the lateral satellites in the $\theta / 2 \theta$ XRD scans (Fig. 4) were fit using a Bede RADS mercury software simulation to determine the composition of the individual SPS layers, and, thus, the degree of intermixing. The composition of the $\operatorname{In}_{x} \mathrm{Al}_{1-x} \mathrm{As}_{\mathrm{s}} / \mathrm{In}_{y} \mathrm{Al}_{1-y} \mathrm{As}$ layers in sample A450 was found to be $x=0.58$ and $y=0.34$, resulting in a mismatch of $1.7 \%$. The fit for sample A500 generated compositions of $x=0.57$ and $y=0.38$, resulting in a mismatch of $1.3 \%$. For sample A520, the compositions were $x$ $=0.58$ and $y=0.35$, resulting in a slightly higher mismatch of $1.6 \%$. Fits for samples grown at even higher temperatures could not be obtained due to disordering of the SPS structures. These results show that the SPS layers are indeed intermixed and that the amount of intermixing decreases as the temperature moves away from the optimum, in agreement with the model for $\ln$ segregation. AlAs/lnAs SPS structures grown at $T<500{ }^{\circ} \mathrm{C}$ are also expected to roughen and to modulate due to decreased In intermixing. However, AlAs/ lnAs SPS samples grown at $T<500{ }^{\circ} \mathrm{C}$ do not exhibit lateral composition modulation, suggesting that the lateral mass transfer that is necessary for composition modulation to develop is inactive at these temperatures for this material system.

For Ga-containing samples, lateral composition modulation is never completely suppressed, however, the least amount of roughening is observed for the sample grown at $T=490^{\circ} \mathrm{C}$. Using this temperature to set the minimum $T$, $E_{1}=2.08 \mathrm{eV}$ is obtained. This value of $E_{1}$ is significantly higher than the binding energy of GaAs. ${ }^{27}$ Fits to determine the composition of the individual SPS layers in this materials system were not possible due to the substantial roughening of SPS interfaces. Nonetheless, these data are in qualitative agreement with the prediction of this model. Namely, that roughening is minimized at some intermediate temperature. At $T<485^{\circ} \mathrm{C}$, the samples formed 3D islands after the critical thickness of the structure was deposited. At $T=490^{\circ} \mathrm{C}$, the samples did not exhibit 3D island formation although some roughening did occur. Therefore, this sample exhibits regular and periodic lateral composition modulation along the whole thickness. Samples grown at $T>500^{\circ} \mathrm{C}$ have very rough interfaces and the lateral composition modulation is irregular.

This model can be used only qualitatively to explain the experimental results because it assumes that surface segregation takes place on a perfectly flat surface. However, these experiments clearly show that roughening occurs, thereby rendering this assumption invalid. Furthermore, this model assumes that only one layer participates in the In exchange process which is unlikely. ${ }^{28}$ As a result, accurate fitting of the parameters to experimental data, especially the values of $E_{s}$, is not possible with the current model. In order to simulate these results more accurately, the composition profiles would need to be calculated by taking exchange interactions between multiple layers and surface features such as steps and islands into account, similar to the work conducted by Braun et al. $^{29}$

\section{CONCLUSIONS}

The microstructure of lateral composition modulation (CM) was compared in two different materials systems, GaAs/InAs and AlAs/InAs SPSs, as a function of the growth temperature. For AlAs/InAs structures deposited at temperatures of $T \leqslant 500{ }^{\circ} \mathrm{C}$ the growth occurs via, layer-by-layer growth mode. No lateral composition modulation was observed by XTEM and the sample had well defined SPS individual layers, i.e., no vertical intermixing. At temperatures of $T>500^{\circ} \mathrm{C}$ some roughening occurs and strong, regular, periodic lateral $\mathrm{CM}$ is observed. The microstructure of lateral composition modulation in GaAs/InAs is significantly different from that of the Al-containing samples. All the samples exhibit some degree of roughness and lateral composition modulation. At low temperatures $\left(T<490{ }^{\circ} \mathrm{C}\right)$ the samples initially have regular, periodic lateral composition modulation which degrades due to island growth. At $T=490^{\circ} \mathrm{C}$, the growth remains nominally planar and the SPSs exhibit strong, regular lateral $\mathrm{CM}$ along the whole thickness. At higher temperatures, 3D islands start to nucleate immediately, the SPS becomes fully disordered, and lateral composition modulation is not correlated from the top to the bottom of the film. Lateral composition modulation appears in both [110] and [1 $1 \overline{0} 0]$ directions for almost all the Ga- and Alcontaining structures. The lateral periodicity $\Lambda$ is approximately $30 \mathrm{~nm}$ and is not considerably temperature dependent. The differences in microstructure between the two material systems can be qualitatively explained using a kinetic model that takes In surface segregation into consideration. These data demonstrate that, at some intermediate temperature, In intermixes into the individual SPS layers, resulting in lower mismatch between the individual layers and more planar growth. At lower and higher temperatures, the mismatch is greater, resulting in more roughening and thus more composition modulation.

\section{ACKNOWLEDGMENTS}

The authors acknowledge helpful discussions with M. Bouville, B. Bennet, R. Twesten, K. Matney, C. Wauchope, A. Huber, B. Orr, and C. Pearson. This work was supported by NSF Grant No. DMR 9973352.

\footnotetext{
${ }^{1}$ A. Zunger and S. Mahajan, Handbook of Semiconductors, edited by T. S. Moss (Elsevier Science, Amsterdam, 1994), Vol. 3, p. 1399.

${ }^{2}$ S. T. Chou, K. C. Hseih, L. J. Chou, and K. Y. Cheng, Appl. Phys. Lett. 66, 2220 (1995)

${ }^{3}$ D. E. Wohlert, K. Y. Cheng, and S. T. Chou, Appl. Phys. Lett. 78, 1047 (2001).

${ }^{4}$ T. Y. Seong and et al., J. Appl. Phys. 73, 8227 (1993).
} 
${ }^{5}$ I. T. Ferguson, A. G. Norman, B. A. Joyce, T. Y. Seong, G. R. Booker, R. H. Thomas, C. C. Philips, and R. A. Stradling, Appl. Phys. Lett. 59, 3324 (1991).

${ }^{6}$ A. G. Norman, T.-Y. Seong, I. T. Ferguson, G. R. Booker, and B. A. Joyce, Semicond. Sci. Technol. 8, S9 (1993).

${ }^{7}$ A. G. Norman et al., Appl. Phys. Lett. 73, 1844 (1998).

${ }^{8}$ J. Mirecki Millunchick, R. D. Twesten, S. R. Lee, D. M. Follstaedt, E. D. Jones, S. P. Ahrenkiel, Y. Zhang, H. M. Cheong, and A. Mascarenhas, J. Electron. Mater. 26, 1049 (1997).

${ }^{9}$ J. Mirecki Millunchick, R. D. Twesten, D. M. Follstaedt, S. R. Lee, E. D. Jones, Y. Zhang, S. P. Ahrenkiel, and A. Mascarenhas, Appl. Phys. Lett. 70, 1402 (1997).

${ }^{10}$ J. Mirecki Millunchick, R. D. Twesten, S. R. Lee, D. M. Follstaedt, E. D. Jones, S. P. Ahrenkiel, Y. Zhang, H. M. Cheong, and A. Mascarenhas, MRS Bull. 22, 38 (1997).

${ }^{11}$ D. E. Wohlert and K. Y. Cheng, Appl. Phys. Lett. 76, 2247 (2000).

${ }^{12}$ R. D. Twesten, D. M. Follstaedt, S. R. Lee, E. D. Jones, J. L. Reno, J. Mirecki Millunchick, A. G. Norman, S. P. Ahrenkiel, and A. Mascarenhas, Phys. Rev. B 60, 13619 (1999).

${ }^{13}$ D. M. Follstaedt, J. L. Reno, E. D. Jones, S. R. Lee, A. G. Norman, H. R. Moutinho, A. Mascarenhas, and R. D. Twesten, Appl. Phys. Lett. 77, 669 (2000).

${ }^{14}$ K. Y. Cheng, K. C. Hsieh, and J. N. Baillargeon, Appl. Phys. Lett. 60, 2892 (1992).

${ }^{15}$ K. Shiraishi, Appl. Phys. Lett. 60, 1363 (1992).

${ }^{16}$ S. T. Chou, K. Y. Cheng, L. J. Chou, and K. C. Hseih, J. Appl. Phys. 78, 6270 (1995).
${ }^{17}$ F. Glas, J. Appl. Phys. 62, 3201 (1987).

${ }^{18}$ D. J. Srolovitz, Acta Metall. 37, 621 (1989); R. J. Asaro and W. A. Tiller, Metall. Trans. 3, 1789 (1972); M. A. Grinfeld, Sov. Phys. Dokl. 31, 831 (1986).

${ }^{19}$ J. E. Guyer and P. W. Voorhees, J. Cryst. Growth 187, 150 (1998); F. Glas, Phys. Rev. B 55, 11277 (1997); F. Leonard and R. C. Desai, ibid. 57, 4805 (1998).

${ }^{20}$ B. J. Spencer, P. W. Voorhess, and J. Tersoff, Phys. Rev. Lett. 84, 2449 (2000).

${ }^{21}$ L. E. Shilkrot, D. J. Srolovitz, and J. Tersoff, Phys. Rev. B 62, 8397 (2000).

${ }^{22}$ C. Dorin, J. Mirecki Millunchick Y. Chen, B. G. Orr, and C. A. Pearson, Appl. Phys. Lett. (in press).

${ }^{23}$ S. R. Lee, J. Mirecki Millunchick, R. D. Twesten, D. M. Follstaedt, J. L. Reno, S. P. Ahrenkiel, and A. G. Norman, J. Mater. Sci. 10, 191 (1999).

${ }^{24}$ T. Shitara, J. H. Neave, and B. A. Joyce, Appl. Phys. Lett. 62, 1658 (1993).

${ }^{25}$ D. M. Follstaedt, S. R. Lee, J. L. Reno, E. D. Jones, R. D. Mascarenhas, and J. Mirecki Millunchick, Mater. Res. Soc. Symp. Proc. 583, 333 (2000)

${ }^{26}$ O. Dehaese, X. Wallart, and F. Mollot, Appl. Phys. Lett. 66, 52 (1995).

${ }^{27}$ J. M. Moison, C. Guille, F. Houzay, F. Barthe, and M. Van Rompay, Phys. Rev. B 40, 6149 (1989).

${ }^{28}$ S. R. Lee, J. L. Reno, and D. M. Follstaedt, Appl. Phys. Lett. 78, 3421 (2001).

${ }^{29}$ W. Braun and H. Ploog, J. Appl. Phys. 75, 1993 (1994). 